\title{
Perfil de cirurgióes-dentistas formados por um currículo integrado em uma instituição de ensino pública brasileira
}

\author{
Aline Claudia Ribeiro Medeiros Silva*, Talissa Mayer Garrido**, Mitsue Fujimaki \\ Hayacibara***, Carina Gisele Costa Bispo***, Rafael Luis da Silva****, Maria Celeste \\ Morita*****, Raquel Sano Suga Terada******
}

\author{
* Mestre em Odontologia Integrada pela Universidade Estadual \\ de Maringá \\ ** Acadêmica do Curso de Odontologia da Universidade Estadual \\ de Maringá \\ *** Professora Adjunta do Departamento de Odontologia da \\ Universidade Estadual de Maringá \\ ***** Bacharel em Sistemas de Informação pela Universidade Paranaense \\ ****** Professora Associada do Departamento de Medicina Oral e \\ Odontologia Infantil da Universidade Estadual de Londrina \\ ******* Professora Associada do Departamento de Odontologia da \\ Universidade Estadual de Maringá
}

\section{RESUMO}

O objetivo deste trabalho foi caracterizar o perfil dos egressos do Curso de Odontologia da Universidade Estadual de Maringá e a contribuição do projeto pedagógico para atuação profissional. Um questionário on-line composto por 20 questões foi enviado via e-mail para os egressos formados entre 2003 e 2010. Para armazenamento e extração das informações utilizou-se o software MYSQL e SQLYOG respectivamente, e foram exportadas para o formato XLS (Microsoft Excel) para a análise descritiva. Do total de 273 egressos deste período, foram contatados $208(76 \%)$ e destes, 148 responderam ao questionário $(71 \%)$. A maioria dos egressos foi do sexo feminino (63\%), faixa etária de 26 a 30 anos, oriundos do Paraná (84\%) e atuava neste estado (80\%). Aproximadamente $50 \%$ apresentaram uma renda anual de 12-36 mil $\mathrm{R} \$$ /ano, a maioria faz ou fez algum curso de pós-graduação (95\%), atuava em consultório particular $(61 \%)$ e apenas $9 \%$ sentiam-se realizados financeiramente. Quanto a proposta pedagógica, mais de $90 \%$ a consideraram boa ou ótima, 59\% relataram que o currículo ocorre parcialmente de forma integrada e que as disciplinas atendiam parcial $(48 \%)$ ou totalmente aos objetivos propostos $(49 \%)$. Concluiu-se que o curso tem for- mado profissionais predominantemente do sexo feminino, jovem e com atuação concentrada no Paraná. O projeto pedagógico do curso tem contribuído para uma formação generalista, as disciplinas atendem aos objetivos do curso, porém na visão dos egressos, o desenvolvimento curricular ocorre de forma parcialmente integrada indicando a necessidade de novas reformulações visando a melhoria do curso.

\section{DESCRITORES}

Recursos Humanos em Saúde. Educação em Odontologia. Avaliação Institucional.

$\mathbf{N}$ as últimas décadas, o exercício da profissão de cirurgião-dentista $(\mathrm{CD})$ tem passado por profundas modificações resultantes da influência de diversos fatores, como as mudanças nas características epidemiológicas da cárie, a crescente demanda por assistência odontológica, a reformulação do sistema de saúde pública e as mudanças socioeconômicas e culturais. ${ }^{1-3}$

O Brasil tem um efetivo de dentistas entre os maiores do mundo. ${ }^{4}$ Ao mesmo tempo, apesar do rápido crescimento do número de egressos da profissão ${ }^{5}$ é um país que ainda apresenta agravos bu- 
Perfil de cirurgiões-dentistas formados por um currículo integrado em uma instituição de ensino pública brasileira • Silva ACRM, Garrido TM, Hayacibara MF, Bispo CGC, Silva RL, Morita MC, Terada RSS

cais e suas sequelas são de grande prevalência, constituindo-se em problemas de saúde pública com importantes consequências sociais e econômicas. ${ }^{6}$ Esta situação paradoxal indica a necessidade de repensar a lógica do trabalho e, consequentemente, a formação dos profissionais que estão exercendo a profissão.

A educação profissional não manteve o ritmo desses desafios, em grande parte devido à fragmentação, desatualização e currículos estáticos que produzem profissionais mal preparados. O redesenho da educação profissional de saúde é emergente e necessário, tendo em vista as oportunidades de mútua aprendizagem e de soluções conjuntas oferecidas pela interdependência global, devido à aceleração dos fluxos de conhecimento, tecnologias, financiamentos e da migração de profissionais e pacientes. ${ }^{7}$

A identificação dos perfis profissionais e estudos de acompanhamento de egressos consiste em uma forma eficiente de organizar o processo de formação profissional e avaliação curricular de uma Instituição de Ensino Superior (IES). ${ }^{5}$ Mesmo com o conhecimento da importância da criação de um sistema de acompanhamento e formação permanente dos alunos, há ainda, um déficit de trabalhos que delineiam o perfil de egressos de um curso voltado à formação de profissionais generalistas, com forte inserção social e um currículo integrado, como ocorre no ensino de graduação em odontologia, incluindo o curso da Universidade Estadual de Maringá.

Na literatura, estudos demonstram mudanças no perfil dos cirurgiões-dentistas e tendências para a profissão. Percebe-se uma progressiva incorporação de tecnologia, de especialização, mudanças visíveis no sistema de saúde público e privado, com redução do exercício liberal estrito e aumento de profissionais com vínculo público, assim como o aumento da escolarização feminina. ${ }^{4,5,8,9}$ Alguns autores apontam para a necessidade de mudanças no ensino odontológico, visto que se faz necessária uma adequação dos recursos humanos formados para a construção de um novo modelo de atenção à saúde, profissionais mais bem preparados para o mercado de trabalho, com uma formação sólida e que corresponda às necessidades da população. ${ }^{8,10-13}$

Desta maneira, a universidade deveria manter um sistema de acompanhamento e formação permanente de seus alunos, que deverá durar até o fim de sua vida profissional, pois uma pesquisa desenvolvida para delinear o perfil dos egressos, na medida em que levanta diagnósticos, apura indicadores de qua- lidade e desenvolve análises sobre a trajetória de seus ex-alunos, subsidia, dessa forma, o planejamento, a adoção e a condução das políticas institucionais relacionadas ao ensino, pesquisa, extensão e gestão da qualidade.

O objetivo do presente trabalho foi caracterizar o perfil dos egressos do Curso de Odontologia da Universidade Estadual de Maringá (UEM) formados entre os anos de 2003 e 2010 e a contribuição do projeto pedagógico do curso para a atuação profissional.

\section{MATERIAL E MÉTODOS}

Todos os procedimentos deste estudo seguiram os princípios éticos estabelecidos pela legislação em vigor e foram aprovados pelo Comitê de Ética em Pesquisa Envolvendo Seres Humanos (CAAE $\mathrm{n}^{\circ}$ 0475.0.093.000-10).

Foram incluídos como sujeitos da pesquisa, acadêmicos que concluíram o curso de graduação entre os anos de 2003 e 2010, formados segundo uma proposta pedagógica baseada em um currículo integrado. Do total de 273 egressos, foram localizados os endereços eletrônicos de 208 egressos (76\% da população do estudo) por meio de consulta aos registros de ex-alunos na Secretaria do Departamento de Odontologia da UEM, contato telefônico ou internet. Portanto, fizeram parte deste estudo um total de 208 egressos.

Aplicou-se um questionário on-line composto por 18 questões fechadas, de múltipla escolha, e 2 questões abertas, divididas em 5 blocos:

I) dados pessoais,

II) perfil socioeconômico e demográfico,

III) campo de atuação,

IV) avaliação pessoal do Projeto Pedagógico e

V) contribuição do Projeto Pedagógico.

O questionário foi enviado via e-mail por meio de um link que ao ser acessado gerava uma página da web contendo o Termo de Consentimento Livre Esclarecido. Somente após leitura e concordância com o mesmo, os egressos tiveram acesso ao questionário. O questionário foi reenviado aos egressos que ainda não haviam respondido, semanas após o primeiro contato.

A estrutura adotada para captação dos dados via website se deu a partir da criação de um formulário baseado no questionário escrito. Para armazenamento dos dados foi utilizado o software de banco de dados MYSQL. Os dados foram armazenados neste software em formato de tabela, onde cada pergunta 
correspondia a uma coluna. As informações foram extraídas utilizando-se o software SQLYOG, que por sua vez apresentou as porcentagens segundo a frequência das respostas. Os resultados foram exportados para o formato XLS (Microsoft Excel) e então procedeu-se a análise descritiva dos dados.

\section{RESULTADOS}

Do total de 208 egressos contatados, 148 responderam ao questionário, o que representa $54 \%$ do total de egressos do curso no período avaliado e $71 \%$ da amostra deste estudo.

Entre os respondentes, 63\% eram mulheres e $37 \%$ homens. Quanto à faixa-etária, 39\% tinham até 25 anos, $51 \%$ de 26 a 30 anos, e $10 \%$ de 31 a 35 anos. A grande maioria dos egressos (84\%) tinham como estado de origem o Paraná, sendo que $80 \%$ do total atuavam neste estado após a conclusão do curso. Observou-se que $7 \%$ vieram de São Paulo, e os demais $(9 \%)$ eram oriundos de diferentes estados como Amazonas, Distrito Federal, Mato grosso, Mato Grosso do Sul, Minas Gerais, Rio de Janeiro e Santa Catarina. Aproximadamente um quinto dos egressos (28 egressos, 19\%) estavam trabalhando em estados diferentes do estado de origem, sendo que $10(7 \%)$ são oriundos de outros estados e permaneceram no Paraná, 12 (8\%) eram do Paraná e foram para outros estados brasileiros, e 6 (4\%) eram de estados como São Paulo, Amazonas e Minas Gerais e foram para outros estados.

A renda anual declarada pode ser observada no Gráfico 1. Verificou-se que aproximadamente metade dos alunos apresentou uma renda na faixa entre 12-36 mil R\$/ano.

A maioria dos egressos (95\%) faz ou fez algum curso de pós-graduação, $47 \%$ cursa ou cursou especialização, $22 \%$ faz ou fez aperfeiçoamento, $20 \%$ estão envolvidos com mestrado, $3 \%$ com doutorado e $3 \%$ com residência.

A distribuição dos egressos do curso de odontologia segundo o vínculo profissional pode ser observada na Tabela 1.

Mesmo tornando-se uma prática em crescimento atualmente, $26 \%$ dos egressos que trabalham atendem para algum convênio ou cooperativa. Do total de respostas, verificou-se que 96 profissionais apresentam apenas 1 vínculo empregatício, 40 profissionais têm dois vínculos empregatícios e 9 deles, 3 vínculos empregatícios. As áreas de atuação profissional mais citadas foram dentística, cirurgia, prótese, periodontia e endodontia, atingindo índices de $28 \%$,

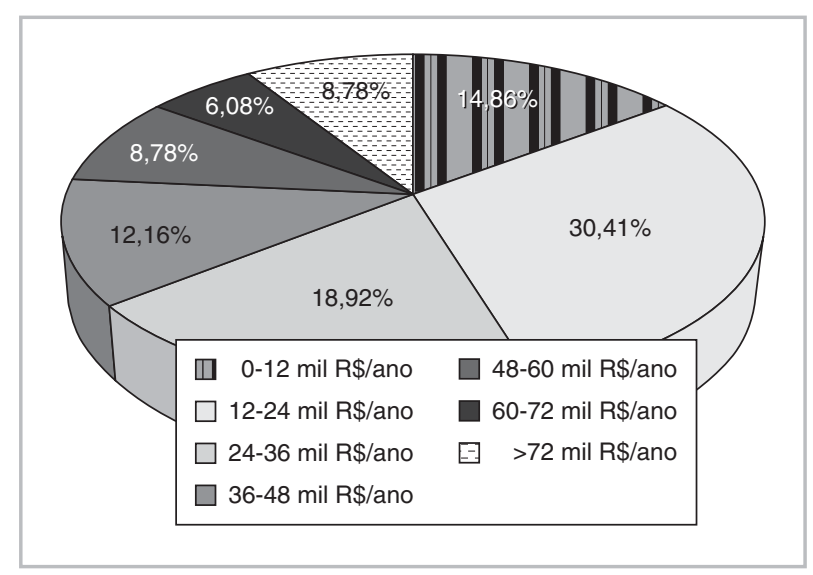

Gráfico 1 - Renda anual dos egressos de 2003 a 2010 da Universidade Estadual de Maringá (\%).

Tabela 1 - Distribuição dos egressos segundo o vínculo profissional.

\begin{tabular}{|l|c|c|}
\hline \multicolumn{1}{|c|}{ Vínculo Profissional } & $\begin{array}{c}\text { Quantidade } \\
\text { de respostas }\end{array}$ & $\begin{array}{c}\% \text { de } \\
\text { egressos }\end{array}$ \\
\hline $\begin{array}{l}\text { Consultório particular de } \\
\text { outro dentista, pagando } \\
\text { porcentagem }\end{array}$ & 76 & 36,5 \\
$\begin{array}{l}\text { Consultório particular } \\
\text { próprio }\end{array}$ & 46 & 22,1 \\
\hline $\begin{array}{l}\text { Serviço público* } \\
\text { Pós-graduação** }\end{array}$ & 40 & 19,2 \\
\hline $\begin{array}{l}\text { Empresa privada, plano de } \\
\text { saúde, sindicatos e } \\
\text { associações }\end{array}$ & 17 & 8,2 \\
\hline $\begin{array}{l}\text { Consultório particular de } \\
\text { outro dentista, pagando } \\
\text { aluguel fixo }\end{array}$ & 5 & 7,7 \\
\hline Não trabalho & 3 & 2,4 \\
\hline Docente & 2 & 1,4 \\
\hline
\end{tabular}

* Rede municipal, ministério da saúde, aeronáutica e hospitais.

${ }^{* *}$ Mestrado, doutorado, residência e estágios.

$19 \%, 11 \%, 11 \%$ e $10 \%$ respectivamente. Quanto à realização financeira, $9 \%$ sentem-se completamente realizados, um pouco mais que a metade, $52 \%$, sentem-se parcialmente realizados, e uma grande parte, $39 \%$ sentem-se pouco realizados. Ainda relacionada a questão financeira, os egressos foram divididos em 2 grupos: os graduados entre 2003 a 2006 (grupo 1) e de 2007 a 2010 (grupo 2). A maior quantidade de profissionais (66\%) que participaram da pesquisa são do grupo 2. Porém, as porcentagens quanto a realização financeira foram bastante semelhantes, com exceção da realização financeira completa, que no grupo 1 era de $14 \%$ e no grupo 2 de $6 \%$ dos egressos. 
Perfil de cirurgiões-dentistas formados por um currículo integrado em uma instituição de ensino pública brasileira • Silva ACRM, Garrido TM, Hayacibara MF, Bispo CGC, Silva RL, Morita MC, Terada RSS

As principais competências e características que os egressos julgaram ser necessárias ao cirurgião-dentistas foram:

- bom relacionamento profissional/paciente,

- visão do paciente como um todo,

- ética,

- capacidade de formular diagnósticos corretos,

- educação permanente, e

- comunicação (Tabela 2).

Tanto em relação ao curso de graduação quanto à proposta pedagógica, mais de $90 \%$ dos alunos os consideraram como bom ou ótimo. Além disso, a maioria $(64 \%)$ também considerou que a proposta curricular contribuiu para uma visão generalista, as-

Tabela 2 - Características/competências necessárias ao cirurgião-dentista.

\begin{tabular}{|l|c|c|}
\hline \multicolumn{1}{|c|}{$\begin{array}{c}\text { Características/ } \\
\text { competências }\end{array}$} & $\begin{array}{c}\text { Total de } \\
\text { respostas }\end{array}$ & $\begin{array}{c}\% \text { de } \\
\text { egressos }\end{array}$ \\
\hline $\begin{array}{l}\text { Bom relacionamento } \\
\text { profissional/paciente }\end{array}$ & 106 & 51,0 \\
\hline $\begin{array}{l}\text { Visão do paciente } \\
\text { como um todo }\end{array}$ & 100 & 48,1 \\
\hline Ética & 93 & 44,7 \\
\hline $\begin{array}{l}\text { Capacidade de formular } \\
\text { diagnósticos corretos }\end{array}$ & 77 & 37,0 \\
\hline $\begin{array}{l}\text { Educação permanente } \\
\text { Comunicação }\end{array}$ & 73 & 35,1 \\
\hline $\begin{array}{l}\text { Capacidade de trabalhar em } \\
\text { equipe }\end{array}$ & 69 & 33,2 \\
\hline $\begin{array}{l}\text { Capacidade de estabelecer } \\
\text { opções de plano de }\end{array}$ & 64 & 31,3 \\
tratamento & 12 & 30,8 \\
\hline Especialização & 56 & 26,9 \\
\hline \begin{tabular}{l} 
Gerenciamento e marketing \\
\hline Tomada de decisão
\end{tabular} & 53 & 25,5 \\
\hline $\begin{array}{l}\text { Atuação generalista } \\
\text { Desenvolvimento de } \\
\text { raciocínio lógico na } \\
\text { formulação de problemas }\end{array}$ & 43 & 20,7 \\
\hline Liderança & 24 & 11,5 \\
\hline
\end{tabular}

sim como $65 \%$ julgou que os professores estimularam a busca por especialização. Por outro lado, $59 \%$ dos alunos relataram que o desenvolvimento do currículo do curso aconteceu de forma parcialmente integrada, sendo que as disciplinas atendiam parcial (48\%) ou totalmente aos objetivos propostos (49\%).

A Tabela 3 apresenta a contribuição do projeto pedagógico para a construção de 5 aspectos fundamentais para o exercício profissional, segundo a visão dos egressos do curso. Verificou-se que mais da metade dos alunos apontou que as disciplinas contribuíram totalmente para a aquisição de competências relacionadas a todos os itens.

\section{DISCUSSÃO}

Do total de cirurgiões-dentistas graduados entre 2003 e 2010, a taxa de retorno dos questionários enviados foi de $71 \%$, superior a outros trabalhos realizados anteriormente. ${ }^{5,8,9} \mathrm{O}$ aumento da taxa de resposta pode ser relacionado à metodologia utilizada, já que o link para o questionário foi fornecido por e-mail.

Neste estudo houve predominância de profissionais do sexo feminino e faixa etária jovem de 26 a 30 anos. Em trabalhos mais antigos, observa-se uma grande quantidade de cirurgiões-dentistas do sexo masculino. ${ }^{8,10,11,14}$ Porém, devido ao ingresso progressivo das mulheres brasileiras no ensino superior a partir da década de 80 , a predominância de cirurgiões-dentistas do sexo feminino pode ser observada desde o final da década de 90 até os dias atuais. ${ }^{4,5,15,16}$

A maioria dos egressos (84\%) são originários do Paraná e após a conclusão do curso, $80 \%$ do total de ex-alunos atuam neste estado, mostrando que houve uma pequena migração para outros estados. A centralização de recursos humanos em Odontologia ocorre também em nível nacional, a distribuição dos CDs brasileiros mostra um quadro de concentração de profissionais em algumas regiões e escassez em outras, observa-se a concentração nas regiões sudeste e sul, onde são menores a proporção de população por CD. ${ }^{4}$ Mostram também que o local de gradu-
Tabela 3 - Contribuição do projeto pedagógico para o exercício profissional (valores em porcentagem do total de respostas).

\begin{tabular}{|l|c|c|c|c|}
\hline \multicolumn{1}{|c|}{ Contribuição } & Sim (\%) & Parcial (\%) & Pouco (\%) & Não (\%) \\
\hline Aspectos sociais, políticos e culturais & 58 & 30 & 11 & 1 \\
\hline Atuação ética & 66 & 26 & 7 & 1 \\
\hline Organização, expressão e comunicação & 55 & 36 & 7 & 2 \\
\hline Raciocínio lógico e análise crítica & 59 & 30 & 10 & 1 \\
\hline Atuação em equipes & 65 & 28 & 6 & 1 \\
\hline
\end{tabular}


Perfil de cirurgiões-dentistas formados por um currículo integrado em uma instituição de ensino pública brasileira • Silva ACRM, Garrido TM, Hayacibara MF, Bispo CGC, Silva RL, Morita MC, Terada RSS

ação tem estreita relação com o local de fixação profissional. Estes dados indicam a necessidade de políticas de incentivo à melhor distribuição da força de trabalho entre as regiões brasileiras, visando a diminuição das desigualdades de acesso aos serviços e ações de saúde. ${ }^{17}$

Atualmente, as carreiras, dentre elas a Odontologia, tornam-se obsoletas em poucos anos se os profissionais não se dedicarem a um permanente processo de aprimoramento de seus conhecimentos. ${ }^{18}$ Em concordância com alguns autores ${ }^{5,9}$ a grande maioria dos egressos (95\%) fazem ou fizeram algum tipo de pós-graduação, entre elas aperfeiçoamento, pós-graduação, mestrado, doutorado, especialização e residência. Esta procura por diversos cursos após a graduação pode estar associada ao acirramento da concorrência, as transformações no mercado de trabalho e ao estímulo que os alunos recebem na graduação.

Corroborando com alguns estudos, ${ }^{5,9,10}$ foi encontrada uma predominância de profissionais trabalhando em consultório particular $(61 \%)$ e aproximadamente $20 \%$ de profissionais atuando no serviço público. Mesmo que a prática privada ainda predomine, há uma tendência à diminuição, visto que ocorre um aumento de profissionais no serviço público como resultado de políticas públicas de saúde e formação de profissionais voltados para o fortalecimento do SUS. As principais transformações com consequências para a prática, perfil profissional e políticas públicas de saúde bucal foi a inserção da Odontologia no Programa de Saúde da Família (2000), homologação das Diretrizes Curriculares Nacionais para os Cursos de Odontologia (2002) e o Programa Brasil Sorridente (2004), que promoveram mudanças no modelo assistencial e na formação da força de trabalho, visando sua inserção no sistema de saúde vigente no país. ${ }^{19-21}$

Quanto à realização financeira, 9\% sentem-se completamente realizados, também foram encontradas altas taxas de insatisfação financeira por outros autores. ${ }^{8,9}$ A comparação entre os alunos formados há mais tempo e os graduados mais recentemente mostrou um índice maior de realização completa nos mais antigos, o que se relaciona com a dificuldade do recém-formado em se estabelecer no mercado de trabalho, devido a alta competitividade, além do fato de que a estabilização na profissão ocorre gradualmente com o passar do tempo.

As áreas mais citadas de atuação em consultório foram Cirurgia, Dentística, Prótese, Periodontia e En- dodontia, o que corrobora com os resultados do trabalho de Arantes et al. ${ }^{22}$ (2009) e é muito semelhante aos resultados de Bastos et al..$^{9}$ (2003). A análise das respostas mostrara que mesmo especializando-se em uma determinada área, os cirurgiões-dentistas continuam atendendo em outras, o que ressalta a importância da formação generalista na Odontologia. Os dados também podem indicar o porquê do interesse pela pós-graduação em disciplinas técnico-científicas ainda ser bem maior que pelas sociais e humanas.

As Diretrizes Curriculares Nacionais (DCN) devem nortear a elaboração dos currículos dos cursos e de seus Projetos Pedagógicos de forma que, ao se graduar, o profissional detenha os conhecimentos e as habilidades e atitudes necessárias ao pleno exercício das suas competências. Segundo as DCN o cirurgião dentista deve ser capaz de realizar as atividades relacionadas à "Atenção à Saúde", "Tomada de Decisões", "Comunicação", "Administração e Gerenciamento", "Educação Permanente" e "Liderança" ${ }^{20}$ Continua indispensável a competência do profissional no domínio de aspectos biológicos e clínicos, entretanto é cada vez mais necessário o desenvolvimento de competências quanto às dimensões ética, política, econômica, cultural e social do seu trabalho, com foco na promoção da saúde em seu sentido integral. ${ }^{23-25}$

Em relação às DCN, as principais competências e características que os egressos julgaram ser necessárias ao cirurgião-dentista foram:

- bom relacionamento entre profissional/paciente,

- visão do paciente como um todo,

- ética,

- capacidade de formular diagnósticos corretos,

- educação permanente e

- comunicação.

A educação permanente pode ser entendida como aprendizagem-trabalho, ou seja, ela acontece no cotidiano das pessoas e das organizações. Ela é feita a partir dos problemas enfrentados na realidade e leva em consideração os conhecimentos e as experiências que as pessoas já têm. Conceito que deve nortear o processo de ensino-aprendizagem e vem sendo incorporado ao Sistema Único em Saúde, por meio da Portaria $n^{\circ}$ 198/GM/MS de 13 de fevereiro de 2004, explicitando a importância da reflexão coletiva da equipe de trabalho, a partir dos problemas reais encontrados na prática cotidiana. ${ }^{26}$ Existe a possibilidade de os ex-alunos confundirem este con- 
Perfil de cirurgiões-dentistas formados por um currículo integrado em uma instituição de ensino pública brasileira • Silva ACRM, Garrido TM, Hayacibara MF, Bispo CGC, Silva RL, Morita MC, Terada RSS

ceito com o fato de estarem realizando pós-graduação ou a considerarem muito importante.

Um fato preocupante é o percentual de respondentes que assinalaram a necessidade de capacidade de gerenciamento e marketing $(6 \%)$ demonstrada pelos alunos. Dean ${ }^{27}$ (1979) constatou que os estudantes não se sentiam capacitados a gerenciar um consultório, principalmente, devido à abordagem deficiente desta disciplina durante o curso de graduação. Costa $e t$ al. ${ }^{8}$ (1992) deixou claro que a faculdade tem sido ineficiente, senão negligente, em atender ao seu caráter profissionalizante no seu ponto mais crítico. Quase 20 anos depois, em tempos de aumento de competitividade, de número de profissionais e de surgimento de novas tecnologias, é possível concluir com base nos resultados que ainda existe esta grande falha na formação.

Em relação ao curso de graduação da UEM, à proposta pedagógica, e a contribuição do currículo integrado para uma visão generalista da profissão, de maneira que as disciplinas atendam aos objetivos do curso. Em novembro de 1991, um novo modelo pedagógico, o currículo multidisciplinar integrado, foi aprovado pelas instâncias superiores da UEM, visando uma formação generalista por meio da integração com as demais áreas do setor de saúde; uma proposta pedagógica inovadora que vai ao encontro do objetivo proposto pelas DCN. Dessa maneira, fica evidente nos resultados deste estudo que o curso se propõe a articular o conhecimento da área odontológica com aspectos sociais, políticos, e culturais da realidade brasileira, desenvolver uma atuação ética com responsabilidade social para construção de uma sociedade includente e solidária em que o profissional tenha organização, expressão, e comunicação do pensamento, realizando raciocínio lógico, análise crítica, compreensão de processos, e desenvolvendo a competência para tomada de decisões, e resolução de problemas no âmbito da área de atuação.

Devido a importância de tais aspectos na formação profissional, são incluídos no Exame Nacional de Desempenho dos Estudantes (ENADE) questões relacionadas com a percepção que o estudante faz referente à contribuição do curso na sua formação profissional e como a instituição formadora influenciou para o desenvolvimento das competências necessárias a sua formação. Assim, a avaliação do ENADE busca elementos de consonância com a DCN, pois além da avaliação do conhecimento técnico-científico necessário para uma boa formação em Odontologia, avaliam-se os cursos com relação às competências preconizadas pelas DCN.

No entanto, observa-se que um pouco mais de $50 \%$ dos egressos consideraram que o desenvolvimento curricular ocorre de forma parcialmente integrada e que os professores estimularam a busca por especialização. Esses dados mostram que apesar do curso objetivar a formação de um profissional generalista ainda persistem resquícios do modelo biomédico sobre o ensino odontológico, características do próprio curso de graduação. A existência de projetos de extensão específicos em determinadas áreas e a própria formação acadêmica dos docentes voltada para um ensino especializado são fatores que também podem contribuir para este estímulo à especialização.

Apesar dos avanços na prática da profissão em consequência da proposta de um novo perfil profissional pela Universidade, ainda existem dificuldades e grandes desafios. Deve-se compreender que a reforma educacional é um processo longo que exige liderança, pactuações coletivas e capacidade de lidar com conflitos para um bom relacionamento entre todos os interessados e, sobretudo, de uma avaliação permanente que retroalimente o processo.

\section{Conclusões}

De acordo com os resultados obtidos neste trabalho, pode-se concluir que o curso de Odontologia da Universidade Estadual de Maringá tem formado profissionais com o perfil predominante do sexo feminino, faixa etária de 26 a 30 anos, com renda anual de 12-36 mil R \$/ano, oriundos do Paraná e parcialmente realizados com a profissão. Observa-se a tendência da centralização de profissionais no Paraná, em consultório particular e da especialização da Odontologia, embora a maioria dos profissionais esteja atuando em diversas áreas da odontologia. O projeto pedagógico do curso tem contribuído para uma formação com visão generalista, as disciplinas atendem aos objetivos do curso, porém, apesar do currículo ser multidisciplinar integrado a maioria dos egressos considerara o desenvolvimento curricular de forma parcialmente integrada. Portanto, observa-se a necessidade de novas reformulações no projeto pedagógico visando cada vez mais a melhoria do curso e consequentemente a concretização do que é preconizado pelas DCN.

\section{ABSTRACT}

Profile of dentists trained with an integrated curriculum at a Brazilian public teaching institution

The aim of this study was to characterize the 
Perfil de cirurgiões-dentistas formados por um currículo integrado em uma instituição de ensino pública

brasileira • Silva ACRM, Garrido TM, Hayacibara MF, Bispo CGC, Silva RL, Morita MC, Terada RSS

profile of the State University of Maringa Dentistry Course alumni and relate the contribution of the institution's pedagogic project to their professional life. An on-line questionnaire composed of 20 questions was sent via email to alumni who graduated between 2003 and 2010. MYSQL and SQLYOG software were used for storage and retrieval of the information, respectively, and data were exported to XLS (Microsoft Excel) format for a descriptive analysis. Of a total of 273 alumni from this period, 208 $(76 \%)$ were contacted, 148 of which responded to the questionnaire $(71 \%)$. The majority of the alumni were women $(63 \%)$, with an age range from 26 to 30 years, who came from Paraná $(84 \%)$ and worked in that state $(80 \%)$. Approximately $50 \%$ reported an annual income of 12 thousand to 36 thousand real ( $\mathrm{R} \$ /$ annum). The majority were currently taking or had taken some graduate courses $(95 \%)$ and were working in private practice $(61 \%)$. Only $9 \%$ felt that they had achieved financial satisfaction. As regards the pedagogic proposal, over $90 \%$ considered it good or excellent, $59 \%$ reported that the curriculum is partially integrated and that the subjects of the curriculum met their proposed objectives either partially $(48 \%)$ or completely (49\%). It was concluded that the course has predominantly trained young, women professionals with activities concentrated in the state of Paraná; the pedagogic project of the course has contributed to fostering a generalist education and that the disciplines have met the objectives of the course; however, from the point of view of the alumni, curricular development is only partially integrated, indicating the need for new formulations with a view to improving the course.

\section{DESCRIPTORES}

Human Resources in Health. Dental Education. Institutional Evaluation.

\section{REFERÊNCIAS}

1. Bravo-Perez M. Inequalities in the workload per dentist in Spain from 1987 to 1997: Workload per dentist. RCOE. 2004; 9: 227-284.

2. Travassos C, Oliveira EXG, Viacava F. Desigualdades geográficas e sociais no acesso aos serviços de saúde no Brasil: 1998 e 2003. Cien Saude Colet. 2006; 11:975-986.

3. Narvai PC, Frazão P, Roncalli AG, Antunes JLF. Dental caries in Brazil: decline, polarization, inequality and social exclusion. Rev Panam Salud Publica. 2006; 19: 385-393.

4. Morita MC, Haddad AE, Araújo ME. Perfil atual e tendências do cirurgião-dentista brasileiro. Maringá: Dental Press; 2010.
5. Nunes MF, Silva ET, Santos LB, Queiroz MG, Leles CR. Profiling alumni of a Brazilian public dental school. Human Resources for Health. 2010; 8: 20.

6. Fernandes LS, Peres MA. Associação entre atenção básica em saúde bucal e indicadores socioeconômicos municipais. Cad Saúde Pública. 2005; 39(6): 930-936.

7. Frenk J, Chen L, Bhutta ZA, Cohen J, et al. Health professionals for a new century: transforming education to strengthen health systems in an interdependent world. The Lancet. 2010; 376: 1923-1958.

8. Costa B, Stegun RC, Todescan R. Realização profissional: uma avaliação entre os dentistas na Grande São Paulo. Rev Assoc Paul Cir Dent. 1992; 46:821-824.

9. Bastos JRM, Aquilante AG, Almeida BS, Lauris JRP, Bijella VT. Análise do perfil profissional de cirurgiões-dentistas graduados na Faculdade de Odontologia de Bauru-USP entre os anos de 1996 e 2000. J Appl Oral Sci. 2003; 11(4): 283-289.

10. Stewart BL, Ralph WJ, Macmillan CH. Survey of dental practice/dental education in Victoria. Part III. Trends in general dental practice. Aust Dent J. 1990; 35:169-180.

11. Falasco RF, Robinson W, Faja BW. Problems encountered by recent graduates in establishing dental practices. J Mich Dent Assoc. 1990; 72:15-19.

12. Feuerwerker LCM. Educação dos profissionais de Saúde hoje - problemas, desafios, perspectivas e as propostas do Ministério da Saúde. Revista da ABENO. 2003; 1:24-27.

13. Morita MC, Kriger L. Mudanças nos cursos de Odontologia e a interação com o SUS. Revista da ABENO. 2004; 1:17- 21.

14. Greenwood LF; Lewis DW, Burgess RC. How competent do our graduates feel? J Dent Educ. 1998; 62: 307-313.

15. Carvalho DR, Carvalho ACP. Motivações e expectativas para o curso e para o exercício da Odontologia. Rev Assoc Paul Cir Dent. 1997; 51 (4):345-349.

16. Rezende FP, Nakanishi FC, Machado ACP, Quirino MRS, Anbinder AL. Perfil, motivações e expectativas dos graduandos e graduados em Odontologia. Revista de Odontologia da Universidade Cidade de São Paulo. 2007; 19(2):165-72.

17. Haddad AE, Morita MC, Pierantoni CR, Brenelli SL, Passarella T, Campos FE. Formação de profissionais de saúde no Brasil: uma análise no período de 1991 a 2008. Rev Saude Publica. 2010; 44: 383-393.

18. Buarque, C. A universidade numa encruzilhada. Brasília: UNESCO; 2003. Acesso em 31/05/2011. Disponível em: http://pages.udesc.br/ f7fsb/Arquivos/universidade.pdf

19. Brasil. Ministério da Saúde. Portaria 1444, de 28 de dezembro de 2000. Dispõe do incentivo financeiro para reorganização da atenção à saúde bucal por meio do Programa Saúde da Família. Diário Oficial da República Federativa do Brasil, Brasília, DF, 29 dez. de 2000, p.85.

20. BRASIL. Ministério da Educação. Conselho Nacional de Educação. Câmara de Educação Superior. Resolução CNE- CES 3, 
Perfil de cirurgiões-dentistas formados por um currículo integrado em uma instituição de ensino pública brasileira • Silva ACRM, Garrido TM, Hayacibara MF, Bispo CGC, Silva RL, Morita MC, Terada RSS

de 19/02/2002. Institui as Diretrizes Curriculares Nacionais do curso de Graduação em Odontologia. Diário Oficial da República Federativa do Brasil, Brasília, 04 de março de 2002, Seção 1, p. 10.

21. BRASIL. Ministério da Saúde. Manual do Brasil Sorridente. Brasília: Ministério da Saúde; 2004. Acesso em 31/05/2011. Disponível em: http://portal.saude.gov.br/portal/saude/ area.cfm?id_area $=406$

22. Arantes ACC, Pinto RS, Ramos TV; Palmier AC. Estágio supervisionado: qual a sua contribuição para a formação do cirurgião-dentista de acordo com as Diretrizes Curriculares Nacionais. Revista de APS. 2009; 12(2):150-160.

23. Moysés SJ. Políticas de saúde e formação de recursos humanos em Odontologia. Revista da ABENO. 2004; 4: 30-37.

24. Mendes RF, Moura MS, Prado Júnior RR, Moura LFAD, Lages
GP, Gonçalves MPR. Contribuição do Estágio Super-visionado da UFPI para formação humanística, social e integrada. Revista da ABENO. 2006; 6:61-65.

25. Garbin CAS, Saliba NA, Moimaz SAS, Santos TS. O papel das universidades na formação de profissionais na área de saúde. Revista da ABENO. 2006; 6:6-10.

26. BRASIL. Ministério da Saúde . Portaria nº 198/GM/MS, de 13 de fevereiro de 2004. Brasília, v.141, n. 32, 2004. Seção 1, p.37-41.

27. Dean, DH. Are dental students prepared to manage their practices? Dent Stud. 1979; 58: 21-22.

Recebido em 08/10/2012

Aceito em 10/12/2012 